\title{
Dynamic Instability of the Stratospheric Polar Vortex*
}

\author{
Isamu Hirota \\ Geophysical Institute, Tokyo University, Tokyo \\ (Manuscript received 20 February 1967, in revised form 23 May 1967)
}

\begin{abstract}
Stability properties of the stratospheric polar vortex deformed under the influence of external forces are examined by use of the barotropic vorticity equation and energy equation.

Time integration of perturbation equation as an initial value problem shows that a barotropic instability corresponding to deformed vortex exists and this instability increases when the vortex is more deformed.

Comparison of stability properties between the northern and southern hemispheric polar vortex is shown by numerical model, and the results of computations show that the northern hemispheric type polar vortex with wave number 2 is more unstable than the southern hemispheric type vortex with wave number 1, and the breakdown is shown for the former case.

Some theoretical considerations are also given concerning the kinetic energy equations for our model. It is found that total kinetic energy much increases when the phase of perturbations is $\mathrm{L} / 4$ ( $\mathrm{L}$ : wave length) different from that of the basic flow.
\end{abstract}

\section{Introduction}

Since the first discovery of the "sudden warming" by Scherhag (1952), synoptic and theoretical studies of the stratospheric waves and their drastic variation associated with the sudden warming have been made extensively.

It is well known that the stratospheric circulation over the polar region in summer is anticyclonic and circular without large scale disturbances, while in wintertime the circumpolar vortex appears and ultra-long waves with wave number one, two or three develop. This "polar-night" vortex is suddenly destroyed, usually in late winter or in early spring and after repetition of this process once or twice the stratospheric circulation becomes anticyclonic.

Fleagle (1958) and Sheppard (1959) proposed baroclinic instability as a mechanism of explosive warming. Murray (1960) investigated two possibilities of breakdown of polar vortex, i.e., breakdown caused by inertia instability and that caused by baroclinic instability,

* Division of Meteorology, Contribution No, 155. and his conclusion is that neither of these instabilities is to be expected in the lower stratosphere. One reason why it is not likely that baroclinic instability is the cause of the breakdown is that static stability in the stratosphere is very large even though vertical wind shear is strong. Then Murray suggested the possibility of barotropic instability. Charney and Stern (1962) investigated a necessary criterion for instability for the special case of an "internal jet", and they further found that criterion is satisfied in the actual atmosphere in wintertime.

Nevertheless, the drastic deformation of the polar vortex has not yet been clearly explained by these instability theories. One reason is because these instability criteria are obtained only for circular, or zonally averaged, flow patterns, whereas the polar vortex in the actual atmosphere is not completely circular but shows rather deformed pattern when instability arises.

Reed et al (1963), Miyakoda (1963), and Murakami (1965) and several others have performed calculations of energy conversions in the lower stratosphere throughout a period that included a sudden warming. In these 
studies, as is customarily done, the energy conversions among the zonal kinetic energy $K_{Z}$, the eddy kinetic energy $K_{E}$, the zonal available potential energy $A_{Z}$ and the eddy available potential energy $A_{E}$ are discussed in some detail.

However, there are some discrepancies in the results of these studies: According to Reed, kinetic energy is transfered, during the period of sudden warming, from eddy to zonal mean flow, while Miyakoda's and Murakami's results show that the transfer of kinetic energy is from zonal to eddy. The latter results suggest that barotropic process might be important in the breakdown phenomenon, although the amount of the converted kinetic energy is not sufficiently large.

Although these partitions of energy may be suitable for describing tropospheric general circulation in which many long waves are embeded in the basically zonal current, there remain some doubts whether similar partition is adequate in the stratosphere.

When we observe the circulation pattern prior to the sudden warming, as mentioned by Miyakoda (1963), the original circumpolar vortex is symmetrically circular and then it starts to elongate. This elongated vortex often persists for several weeks in mid-winter, and then the breakdown of the vortex occurs and is sometimes accompanied by the sudden warming. Thus we shall distinguish two phases in the process of stratospheric circulation, i.e., the elongation of the polar vortex and the following breakdown.

During the first phase, the circumpolar flow has a pattern of so-called ultra-long waves of wave number one or two and this flow pattern may be regarded as the basic current. In this period, $K_{E}$ as usually defined increases. However, this increase of $K_{E}$ does not necessarily correspond to the breakdown of the vortex. We consider that the mechanism which is directly responsible to the breakdown should be sought as a kind of instability of this elongated basic flow. Thus the definition of "eddy" with respect to the stratosphere circulation must be different from the ordinary one. The elongation of the polar vortex in wintertime, therefore, is considered to be one of the necessary conditions for instability. From this point of view, in a previous paper, Matsuno and Hirota (1966) carried out numerical calculations of the breakdown of the polar vortex in the 1958 case.

In the present paper we shall deal with this kind of instability by use of the model polar vortex which is constructed so as to simulate the typical configuration of the actual polar vortex in winter.

The main purposes of this study are as follows ;

(a) To investigate stability properties of the deformed vortex and to obtain the relationship between the instability criterion and the extent of elongation.

(b) To compare the stability of an elongated type vortex with that of an eccentric type vortex. This is to compare the dynamics of the northern hemispheric polar vortex with that of the southern hemispheric one.

(c) To investigate the nature of the instability theoretically and to discuss the instability from the kinetic energy aspect.

\section{Basic assumptions}

Let us first consider perturbation superposed upon a quasi-steady elongated circumpolar flow.

The equation of motion for this steady basic flow may be written as

$$
\boldsymbol{V} \cdot \boldsymbol{\nabla} \boldsymbol{V}+f \boldsymbol{k} \times \boldsymbol{V}+\nabla \phi=\boldsymbol{F}
$$

where $\boldsymbol{V}$ denotes two-dimensional velocity of the basic flow, $f$ Coriolis parameter, $k$ unit vector of vertical direction, $\phi$ geopotential, and $\boldsymbol{F}$ means external forcing function.

The physical meaning of the external forcing function $\boldsymbol{F}$ is interpreted as follows: Although we assume that the basic flow can be approximated as two-dimensional flow, the elongated polar vortex must be maintained baroclinically or by the effect of the large scale heating or cooling. Then the left hand side of the equation (2.1) is not equal zero, but balanced with several quantities including vertical velocity' and the divergent part of horizontal velocity.

On the contrary, perturbations are assumed to be free from the external forces, and thus we have the perturbation equation of motion 
as

$$
\frac{\partial}{\partial t} \boldsymbol{v}^{\prime}+\boldsymbol{V} \cdot \nabla \boldsymbol{v}^{\prime}+\boldsymbol{v}^{\prime} \cdot \nabla \boldsymbol{V}+\boldsymbol{v}^{\prime} \cdot \Delta \boldsymbol{v}^{\prime}+f \boldsymbol{k} \times \boldsymbol{v}^{\prime}+\nabla \phi^{\prime}=0
$$

where notation prime' means perturbation.

Taking the rotation of (2.2), we have the perturbation vorticity equation as

$$
\frac{\partial}{\partial t} \zeta^{\prime}+V \cdot \nabla \zeta^{\prime}+v^{\prime} \cdot \nabla\left(\eta+\zeta^{\prime}\right)=0
$$

where $\zeta^{\prime}$ denotes perturbation vorticity and $\eta$ absolute vorticity of the basic field.

Now, the quantities concerning the basic flow are divided into two parts, i.e., zonally averaged part which is denoted by-and the elongated basic flow part denoted by $\sim$. The vorticity equation (2.3) is thus rewritten as

$$
\frac{\partial}{\partial t} \zeta^{\prime}+(\overline{\boldsymbol{V}}+\tilde{\boldsymbol{V}}) \cdot \nabla \zeta^{\prime}+\boldsymbol{v}^{\prime} \cdot \nabla\left(\bar{\zeta}+\tilde{\zeta}+f+\zeta^{\prime}\right)=0 .
$$

On the other hand, in the usual treatment, the basic current is assumed to be the function of $y$-direction only, that is,

$$
\frac{\partial}{\partial t} \zeta^{\prime}+\left(\bar{U}+u^{\prime}\right) \frac{\partial}{\partial x} \zeta^{\prime}+v^{\prime} \frac{\partial}{\partial y}\left(\bar{\zeta}+f+\zeta^{\prime}\right)=0 .
$$

Compared with the equation (2.5), the equation (2.4) has somewhat different features, especially in the effect of the heterogeneous distribution of vorticity, $\boldsymbol{v}^{\prime} \cdot \nabla \tilde{\zeta}$, and horizontal advection caused by the standing wave, $\tilde{\boldsymbol{V}} \cdot \nabla \zeta^{\prime}$.

The equation (2.4) can be rewritten in Jacobian form by use of the stream function $\phi, \quad(\boldsymbol{V}=\boldsymbol{k} \times \nabla \psi)$ as

$$
\begin{gathered}
\frac{\partial}{\partial t} \nabla^{2} \psi^{\prime}+J\left(\psi_{0}, \nabla^{2} \psi^{\prime}\right)+J\left(\phi^{\prime}, \nabla^{2} \psi_{0}+f\right) \\
+J\left(\phi^{\prime}, \nabla^{2} \phi^{\prime}\right)=0
\end{gathered}
$$

where the subscript 0 denotes the basic current including the zonal part and the elongated part.

It should be noted that the non-linear term $J\left(\psi^{\prime}, \nabla^{2} \psi^{\prime}\right)$, is included in (2.6) for the purpose of determination of the sign of disturbance.

Our problem is to solve (2.6) and to investigate the condition for the growth of the perturbation. The similar but different case, where the elongated basic flow is missing, was treated by Kuo (1949), who obtained the instability criterion analytically. Even in this case it is not easy to handle it analytically. Our case is even more difficult to do so. We shall, therefore, try to solve it numerically.

Time integrations are carried out with use of the finite difference form of the perturbation equation (2.6).

A Cartesian coordinate grid system including $25 \times 25$ grid points is used. The grid size $\Delta x$ is about $550 \mathrm{~km}$.

This grid system covers the hemisphere north of $23^{\circ} \mathrm{N}$ (or S) latitude.

As was discussed in the previous paper (Matsuno and Hirota ; 1966) it can be expected that a most preferred wave will appear in carrying out the time integration of the perturbation equation (2.6) starting with an arbitary distribution of the perturbation $\psi^{\prime}$. The pattern thus selected can be regarded as a manifestation of instability for a given elongated basic current.

\section{Basic flow in numerical model}

In order to construct a circular polar vortex

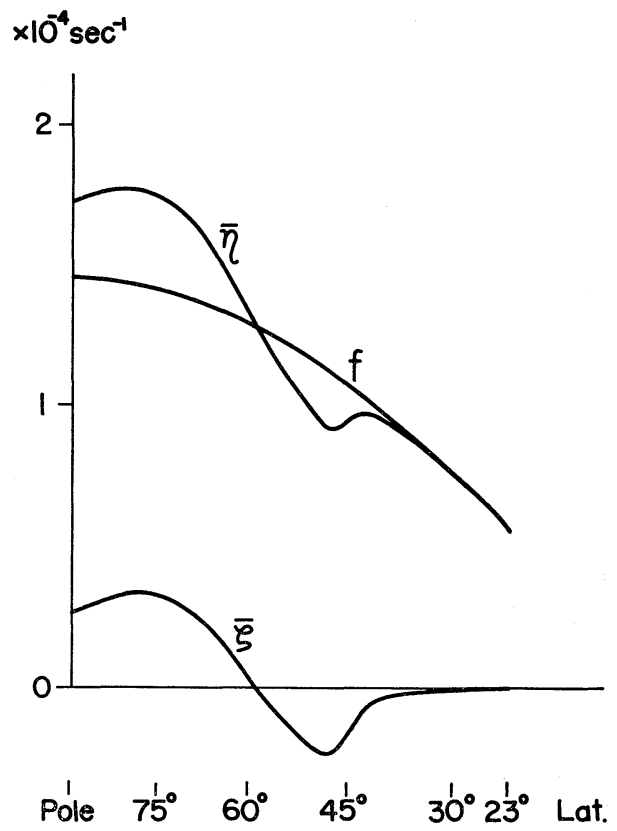

Fig. 1. Latitudinal variations of relative vorticity $\bar{\zeta}$, Coriolis factor $f$ and absolute vorticity $\eta$. 
in numerical model, we determined the radial distribution of relative vorticity $\zeta(r)$. In Fig. $1, \zeta(r)$ is shown which is taken from the zonal mean values of relative vorticity at the $30 \mathrm{mb}$ level computed by Miyakoda (1963) as the monthly mean value of midwinter (1958). Corioli's parameter $f(r)$ and absolute vorticity $\bar{\eta}(r)$ are also shown Fig. 1.

The stream function $\bar{\phi}$ corresponding to circular symmetric vortex is computed by the formula

$$
\nabla^{2} \bar{\phi}=\bar{\zeta}
$$

and the zonal wind velocity is given by

$$
\bar{V}_{\theta}=\frac{\partial \bar{\phi}}{\partial r}
$$

where $r$ denotes the distance from the Pole and $\theta$ is azimuth.

The wind profile thus computed is shown in Fig. 2. This wind velocity has a maximum at about $65^{\circ} \mathrm{N}$ (or $\mathrm{S}$ ) and the maximum velocity is about $35 \mathrm{~m} / \mathrm{sec}$.

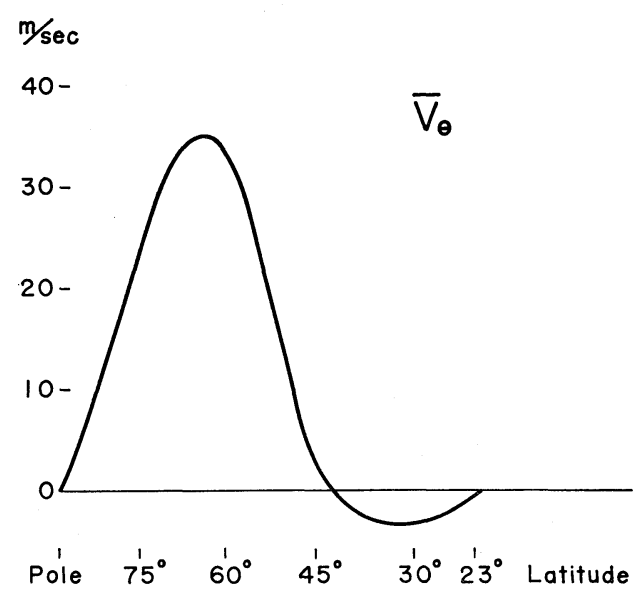

Fig. 2. Mean zonal velocity profile computed from $\bar{\zeta}$.

Next, concerning the deformation of the vortex, we have to determine the functional from of the deformed part of basic stream function, $\tilde{\phi}(r)$.

In order to obtain a relationship between the deformed part and the zonal mean part of the basic currents, we have constructed a radial distribution of the deviation of the isobaric height $\Delta Z$ from the zonal average based on the observations at $30 \mathrm{mb}$ on Janu- ary 25, 1958.

$\Delta Z(r)=Z(r)-\bar{Z}(r)$ is taken along the trough line of the elongated vortex. The amplitude of $\Delta Z(r)$ is shown in Fig. 3.

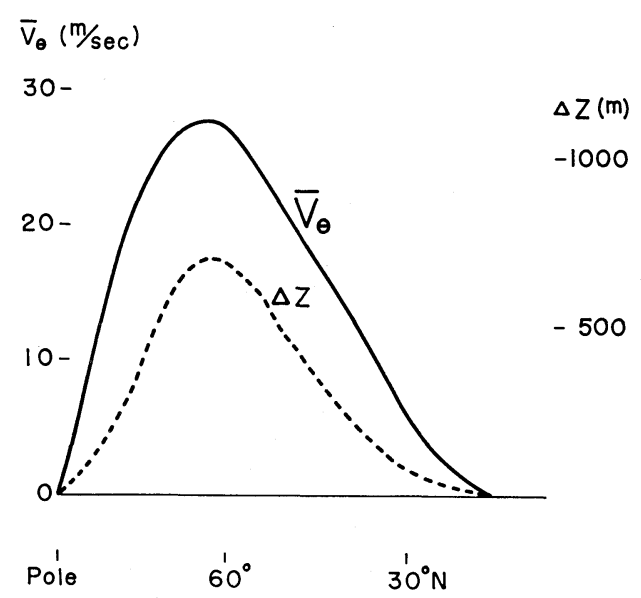

Fig. 3. Mean zonal velocity $\bar{V}_{0}$ and deviation of height $\Delta Z$ observed in $30 \mathrm{mb}$ Jan. 25, 1958.

Compared with the zonal mean velocity profile shown in the same figure, the deformation part is nearly proportional to the mean zonal velocity.

Therefore, in the following, we assume

$$
\tilde{\phi}(r)=a \frac{\partial \bar{\phi}}{\partial r}
$$

where $a$ may be considered as a measure of the amplitude of the elongation.

The entire form of the deformed part of the stream function is given by

$$
\tilde{\phi}(r, \theta)=\tilde{\phi}(r) \sin k \theta
$$

where $k$ denotes wave number.

The wave number $k$ and $a$ are important parameter which determine the stability property of the elongated basic current.

Superposing the deformed part on the circular symmetric polar vortex, we obtain the expression for the total elongated vortex as

$$
\phi_{0}(r, \theta: a, k)=\bar{\phi}(r)+a \frac{\partial \bar{\phi}}{\partial r} \sin k \theta
$$

An example of the elongated vortex thus produced, in the case of $k=2$ and $a=1$, is shown in Fig. 4. This pattern resembles the 


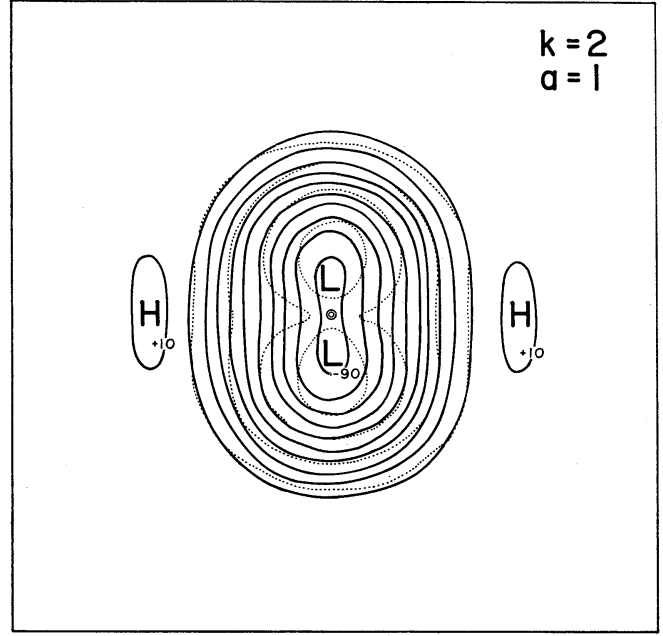

Fig. 4. Elongated polar vortex in the case of $k=2$ and $a=1$. Full line is the stream function and dotted line is the absolute vorticity.

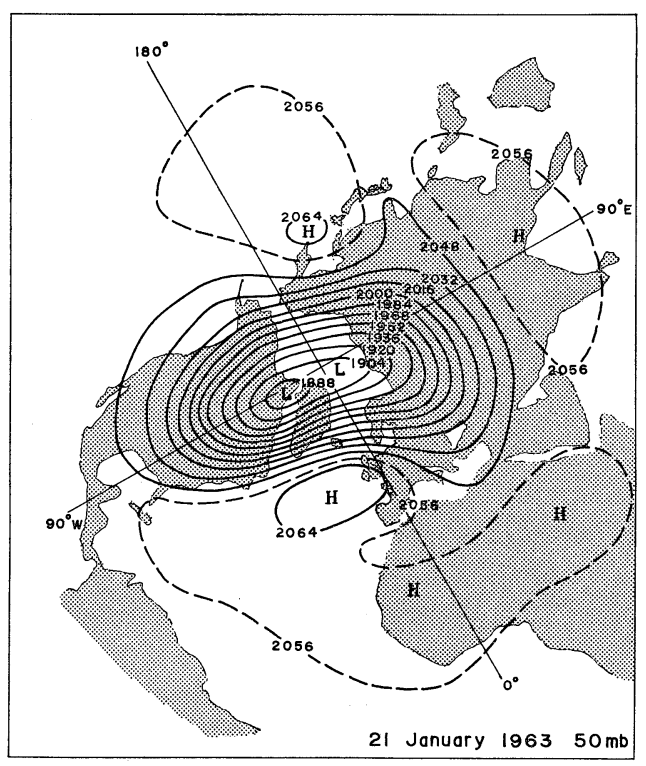

Fig. 5. Observed flow pattern of an elongated polar vortex. (after Scherhag)

flow pattern in the northern hemispheric stratosphere in wintertime, In Fig. 5, the observed flow pattern at the $50 \mathrm{mb}$ level is shown, as an example of the flow pattern just before the occurrence of breakdown phenomenon.

4. Stability property as related to the amplitude of deformation

First, numerical tests were made using the perturbation equation (2.6) for the purpose of investigating the relationship between the instability and the amplitude of deformation $a$. The wave number $k$ of the basic flow is taken to be 2 for the sake of simplicity.

The case of $k=1$ is also computed, and will be discussed in section 6 .

The pattern of the initial perturbation stream function adopted in this test was constructed with the harmonics of wave number 1 and 2 . In order to investigate the stability of the basic flow, we shall show the time change of kinetic energy of the perturbation in the whole domain rather than the time evolution of horizontal pattern of perturbations.

The total kinetic energy $K^{*}$ can be divided into three parts as

$$
K^{*}=\bar{K}+\widetilde{K}+K^{\prime}
$$

where $\quad \bar{K}=\frac{1}{2} \boldsymbol{V}^{2}, \widetilde{K}=\boldsymbol{V} \cdot \boldsymbol{v} \quad$ and $\quad K^{\prime}=\frac{1}{2} \boldsymbol{v}^{2}$.

Since we have assumed that the basic flow is in steady state, kinetic energy of the basic flow $\bar{K}$ does not vary with time, i.e., $\partial / \partial t \bar{K}$ $\equiv 0$, and when there is no external force, total kinetic energy $K^{*}$ must be conserved too, then the energy exchange process is represented as $\tilde{K}+K^{\prime}=$ const. On the other hand, when there is some external forces, $K^{*}$ itself may vary with time.

In this section, however, we confine ourselves only to show time change of $K^{\prime}$. More detailed discussion about the time change of kinetic energy $K^{*}, \tilde{K}$, and $K^{\prime}$ will be shown later in section 7 .

The results are summarized as follows; (i) The case $a=0$

When $a$ is zero, the basic flow pattern is circular and symmetric. In this case the instability of the basic flow can be described by ordinary Rayleigh's criterion. As is shown in Fig. 1, the space derivative of absolute vorticity with respect to $r$, i.e., $\partial \bar{\eta} / \partial r$, changes its sign at around $75^{\circ}$ latitude, and some eigensolutions with positive growth rate may exist.

The most unstable wave corresponding to this circular vortex is shown to be of wave number 3. Actually in the course of time integration, the perturbation with wave num- 
ber 3 appeared.

Since total kinetic energy is conserved in the case of no external force, the kinetic energy of the disturbance i.e.,

$$
<K^{\prime}>\equiv \iint \frac{1}{2} \boldsymbol{v}^{2} d x d y
$$

oscillates with time as is shown in Fig. 6. $A$ disturbance of this type does not grow exponentially.

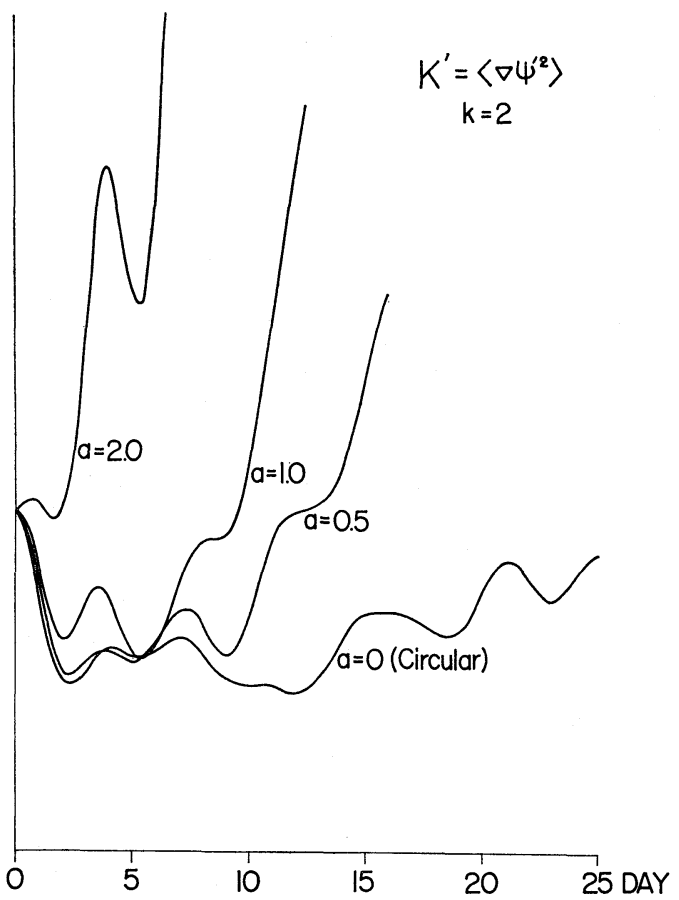

Fig. 6. Time changes of kinetic energy of the disturbances $K^{\prime}$.

This result suggests that barotropic instability for a purely circular vortex is weak and unlikely to explain the drastic breakdown of the polar vortex in the actual winter stratosphere.

(ii) The case $a=0.5,1.0,2.0$

On the other hand, when the circumpolar vortex is elongated, the stability property of the vortex differes from that of the circular symmetric vortex.

As is shown in Fig. 6, the kinetic energy of the disturbances introduced in the elongated basic flow grow almost exponentially with time. The larger the amplitude of elongation $a$, the more rapidly $K^{\prime}$ grows.
Moreover, these three curves in this figure show similar features in their time changes. This means that the behavior of the preferred perturbations with maximum growth rate in these three cases are similar to each other. This fact is also proved by time evolutions of disturbances in these three cases, which are not presented here.

From these computations, it may be concluded that the barotropic instability due to the deformed basic flow is quite different from the ordinary Rayleigh type instability, and this instability becomes large as the basic flow deforms.

It should be noted that the instability mechanism due to the deformation of the basic flow would be hindered if we take the zonally averaged flow as the basic current.

\section{Initial conditions for disturbances}

In section 2, we assumed that the most preferred perturbation for a given basic current can be obtained by the time integration of (2.6) as an initial value problem. We shall next investigate whether or not the perturbation growth depends upon the choice of the initial perturbation.

Diving first to the conclusion, any initial spectral distribution of the perturbations is eventually organized into a systematic wave of wave number 2, due to the instability of this kind. Of course this type of selective development of disturbance is quite usual in any instability problem.

In order to show this the "random distribution" of perturbation stream function is used as an initial condition.

In practice, the initial condition shown in Fig. $7 \mathrm{a}$ is computed with the use of a random number at each grid point and is confined to the middle and higher latitudes. Hatched regions in Fig. 7a have positive values of $\phi^{\prime}$, and the others have negative values. Maximum value of $\phi^{\prime}$ is set to be about $2 \%$ of the maximum amplitude of $\bar{\phi}$.

Starting with this initial condition, the time integration of equation (2.6) is carried out. The basic flow pattern is the case of $k=2$ and $a=1$ (shown in Fig. 4). The time evolution of disturbances is shown in Fig. $7 \mathrm{~b}$ -Fig. 7d.

At first, the perturbation with wave num- 


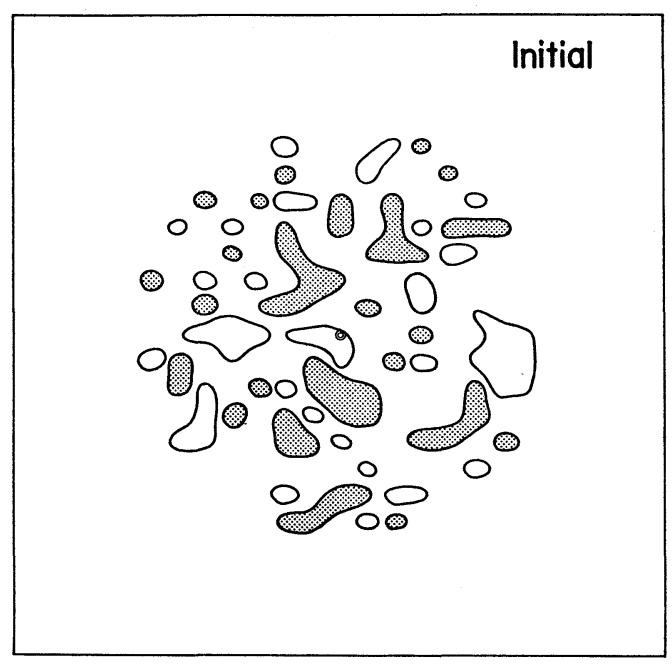

(a)

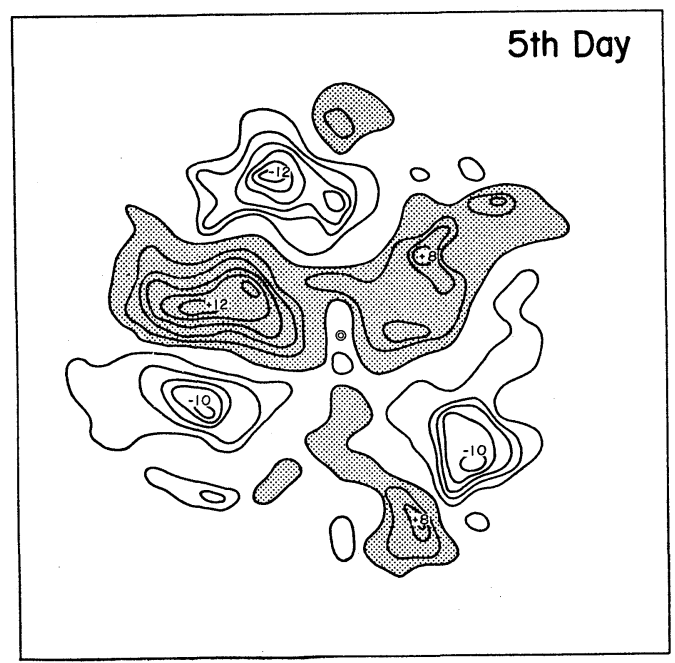

(c)

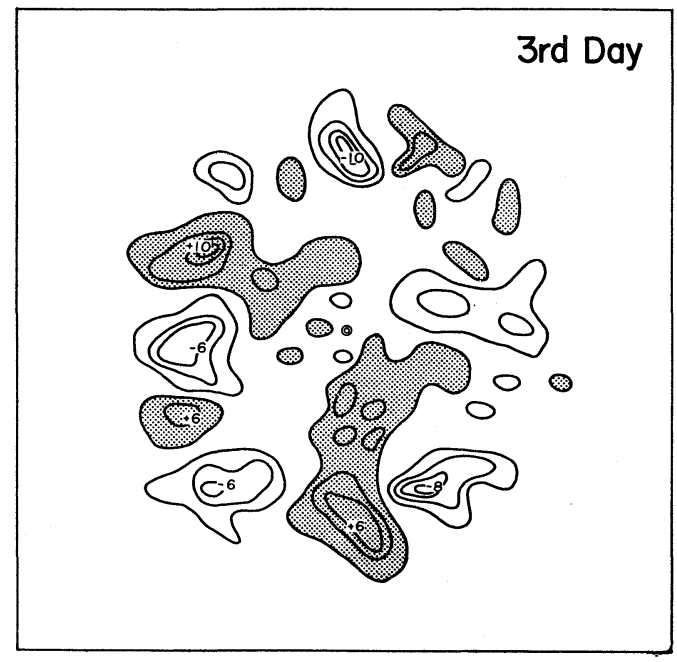

(b)

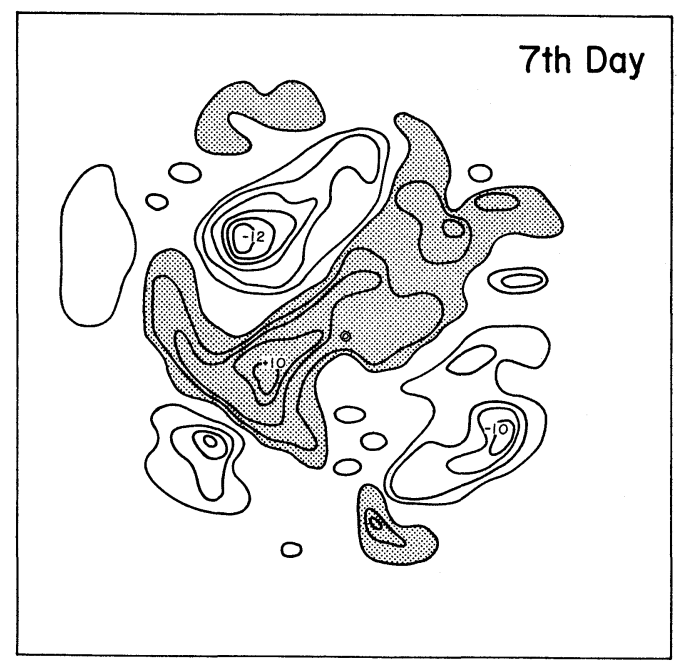

(d)

Fig. 7. Initial pattern of the disturbance $\phi^{\prime}$, and its time evolutions.

ber 3 tends to grow as is shown in Fig. $7 \mathrm{~b}$ Fig. 7c. This may be a manifestation of the eigensolution whose wave number is 3 for this circular vortex.

The disturbance with wave number 2 developed with time, when the deformed basic flow $(k=2, \quad a=1)$ is used. As was discussed in the previous section, this wave is more unstable than that of wave number 3 , then it becomes predominant in the whole domain.

It is seen from Fig. $7 \mathrm{~d}$ that the polar region is covered by positive disturbance and negative maxima appear in outer region.
The pattern thus selected has a tendency to intensify the elongation of polar vortex and therefore the breakdown can be expected from these unstable waves, although their amplitudes are not yet so large.

\section{Comparison of stability properties be-} tween two types of deformed vortices

In the northern hemispheric stratosphere, the most prevailing ultra-long wave in wintertime is of wave number 2, which is due to mainly the large scale topographical effect and differential heating over the two continents and two oceans. 


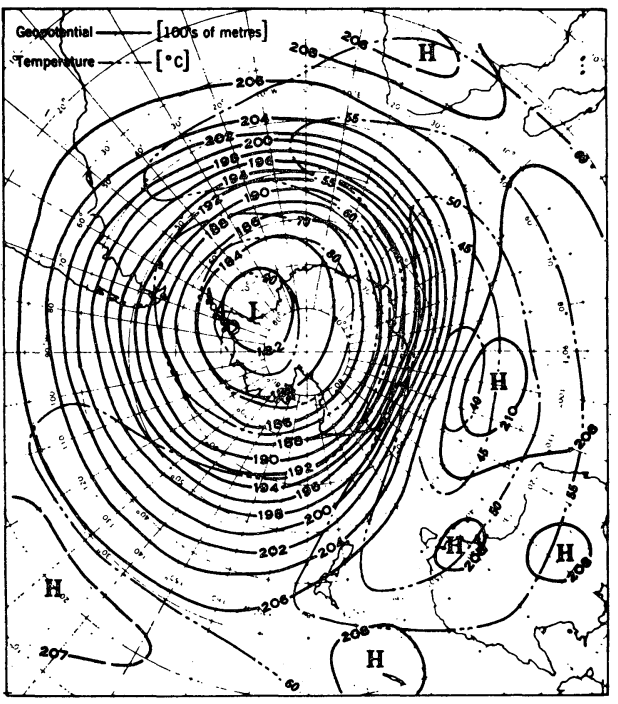

Fig. 8. Observed flow pattern over the southern hemisphere at $50 \mathrm{mb}, 24$ August, 1964. (after Zudanov)

On the other hand, the polar vortex in the southern hemisphere is nearly circular, probably due to the simpler surface topography of the Antarctica.

In Fig. 8 is shown an example of the flow pattern at $50 \mathrm{mb}$ over the antarctic region given by Zudanov (1965). According to his analyses, the following features are described as the behavior of the polar vortex in the antarctic region.

(i) The polar vortex does not suddenly breakdown, but the center of the vortex is pushed away from the South Pole in late winter or in early spring, thus causing the appearance of wave number 1 .

(ii) Although the sudden warming phenomenon is observed in the stratosphere over the Antarctica, it is not as drastic as that of the northern hemisphere.

Thus numerical calculations based on our model are carried out in order to compare the difference of characteristic features of dynamical stability between the polar vortex of wave number one and two.

First of all, a simple theoretical considerations should be given concerning the horizontal distribution of absolute vorticity for these two types of model vortex.

As we have assumed previously, the amplitude of deformation of basic flow is propor- tional to the zonal velocity profile, and the deformation has a sinusoidal distribution with wave number $k$ around the latitude circle, i.e., $\tilde{\phi}=a(\partial \bar{\phi} / \partial r) \sin k \theta$.

The radial distribution of absolute vorticity $\eta(r)$ is written by

$$
\eta(r)=f+\bar{\zeta}(r)+\tilde{\zeta}(r)
$$

and

$$
\begin{aligned}
\frac{\partial \eta}{\partial r}= & \frac{\partial \bar{\zeta}}{\partial r}-\beta+\left\{\frac{\partial^{2} \bar{\zeta}}{\partial r^{2}}+\frac{1-k^{2}}{r^{2}} \bar{\zeta}\right. \\
& \left.-\frac{3\left(1-k^{2}\right)}{r^{3}} \frac{\partial \bar{\psi}}{\partial r}\right\} a \sin k \theta
\end{aligned}
$$

where $\bar{\zeta}$ denotes the relative vorticity with respect to circular vortex, $\beta=-\partial f / \partial r$ latitudinal variation of Corioli's factor, and the third term on the right hand in Eq. (6.2) comes from the deformation part.

Now, let us consider the case in which the ordinary Rayleigh's criterion for barotropic instability is not satisfied, that is, $\partial \bar{\zeta} / \partial r-\beta$ does not change its sign. Nevertheless, because of the third term in Eq. (6.2), Rayleigh's criterion may be satisfied in particular meridional cross sections. It is also easily seen from the experssion (6.2) that the case of $k=1$ has a special feature.

In Fig. 9 are shown three curves of $\partial \eta / \partial r$ corresponding to a circular vortex and deformed vortex of $k=1$ and $k=2$ where the parameter $a \sin k \theta$ is taken to be unity. The value of $\partial \eta / \partial r$ changes its sign in middle latitudes for $k=2$. From these points of view, roughly speaking, it is expected that the deformed vortex of wave number 2 may be dynamically unstable more than the other,

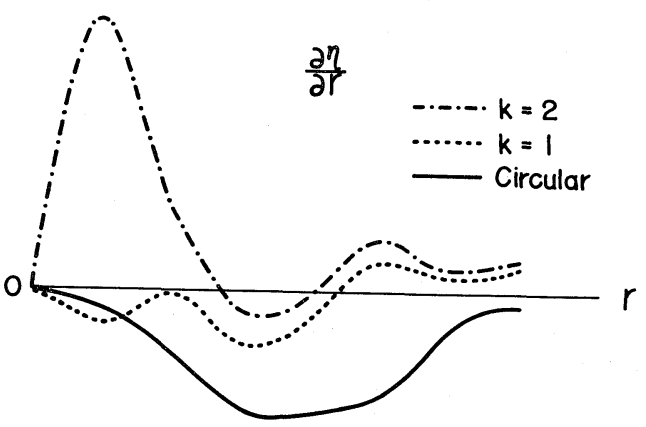

Fig. 9. Radial distribution of $\partial r / \partial r . \quad a \sin k \theta$ is taken to be unity. 
other, although Rayleigh's criterion is not the exact measure for instability in our problem.

The basic flow pattern in the case of $k=1$ $a=2$ is shown in Fig. 10. Long time integration is carried out by using the same initial condition for perturbation with that shown in Fig. 7a.

The perturbation gradually grows during the course of numerical time integration. The perturbation stream function at the 10th day is shown in Fig. 11.

Further numerical integrations were carried out for several cases with various parameters $k$ and $a$.

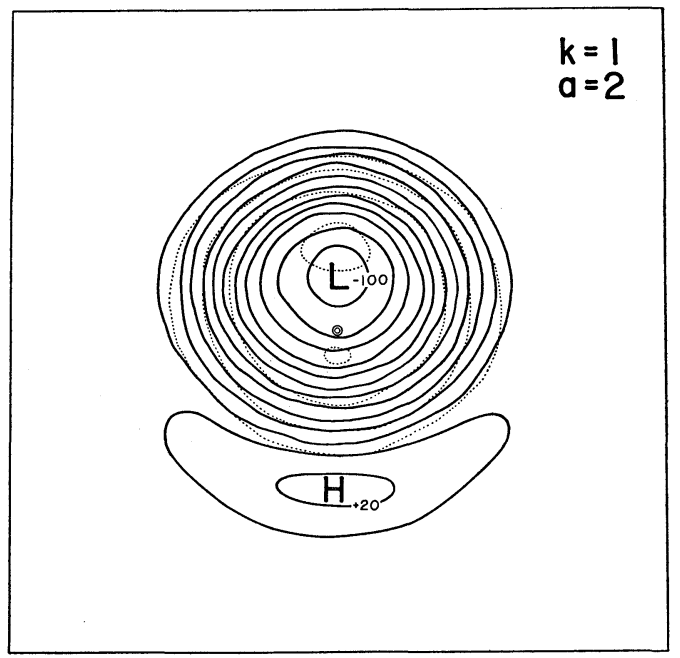

Fig. 10. Asymmetrical polar vortex in the case of $k=1$ and $a=2$.

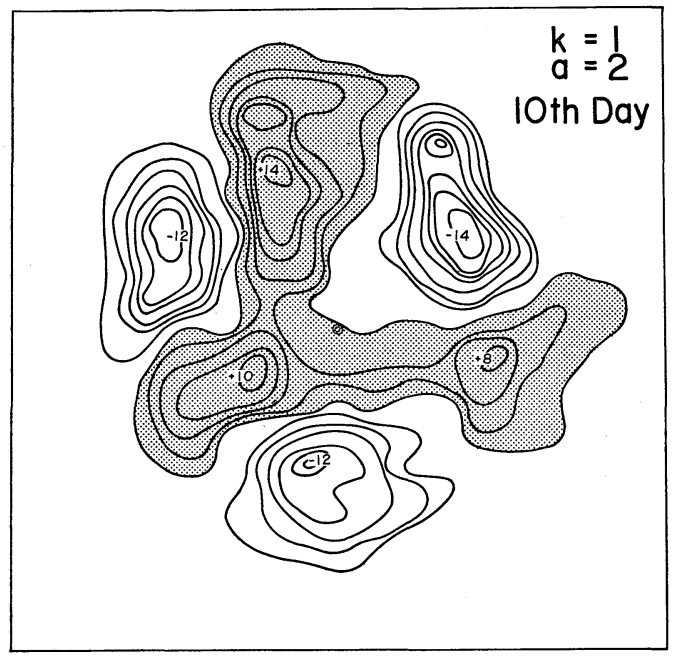

Fig. 11. Disturbances corresponding to a basic flow shown in Fig. 10.

$$
K^{\prime}=\left\langle\nabla \psi^{\prime 2}\right\rangle
$$

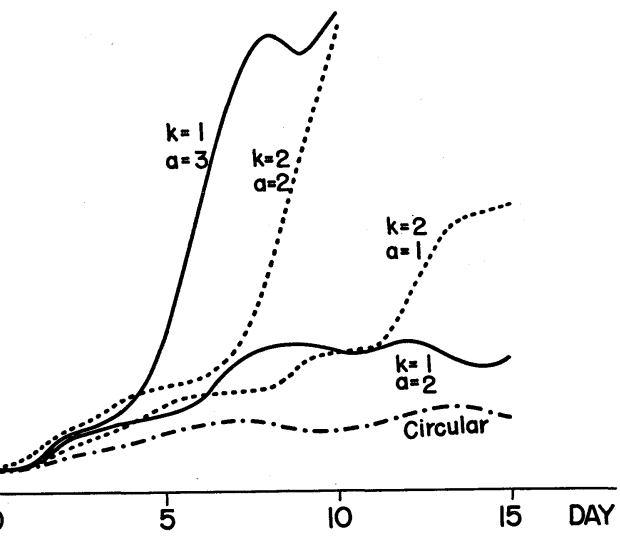

Fig. 12. Time changes of kinetic energy of disturbances $K^{\prime}$.

The time change of perturbation kinetic energy for these cases are shown in Fig. 12.

The results are summarized as follows;

(i) When $a$ is kept constant, the growth rate of disturbance for $k=2$ is greater than that for $k=1$.

(ii) In the case of $k=1$ and $a=2, K^{\prime}$ does not grow rapidly but shows an oscillation similar to the case of circular vortex, while $K^{\prime}$ grows exponentially in the case of $k=2$ and $a=2$.

(iii) Although $K^{\prime}$ becomes large in the case of $k=1$ and $a=3$, the breakdown does not occur as shown in discussions of section 7 .

From these computations, it is concluded that the basic flow with wave number 2 is dynamically more unstable compared with that with wave number 1 . These results are qualitatively in agreement with observed behavior of the polar vortex of the northern and southern hemisphere.

\section{Energy equations}

Although the kinetic energy of the disturbances has been taken into consideration in section 4 for the purpose of detecting instability, it is not sufficient since there can exist not only the pure exchange of kinetic energy between zonal current and disturbance but also the inflow of kinetic energy into the system because of the external forces. 
Thus the energy equations for the whole system should be formulated.

The perturbation equation of motion (2.2) is rewritten as

$$
\begin{gathered}
\frac{\partial u}{\partial t}+U \frac{\partial u}{\partial x}+V \frac{\partial u}{\partial y}+u \frac{\partial}{\partial x}(U+u) \\
+v \frac{\partial}{\partial y}(U+u)-f v=-\frac{\partial \phi^{\prime}}{\partial x}
\end{gathered}
$$

where $U$ and $V$, the velocities of basic flow, are assumed to be non-divergent. Similarily we have

$$
\begin{gathered}
\frac{\partial v}{\partial t}+U \frac{\partial v}{\partial x}+V \frac{\partial v}{\partial y}+u \frac{\partial}{\partial x}(V+v) \\
+v \frac{\partial}{\partial y}(V+v)+f u=-\frac{\partial \phi^{\prime}}{\partial y}
\end{gathered}
$$

By use of the notations

$$
U^{*}=U+u, \quad V^{*}=V+v,
$$

the time change of total kinetic energy $K^{*}$ is written as

$$
\begin{aligned}
\frac{\partial}{\partial t} K^{*}= & \frac{\partial}{\partial t}\left[\frac{1}{2}\left(U^{* 2}+V^{* 2}\right)\right]=\frac{\partial}{\partial t}\left[\frac{1}{2}\left(u^{2}+v^{2}\right)\right] \\
& +\frac{\partial}{\partial t}[U u+V v]=\frac{\partial}{\partial t} K^{\prime}+\frac{\partial}{\partial t} \tilde{K}
\end{aligned}
$$

where

$$
K^{\prime}=\frac{1}{2}\left(u^{2}+v^{2}\right), \quad \tilde{K}=U u+V v .
$$

Making of $u \times(7.1)+v \times(7.2)$, and keeping $\boldsymbol{V} \cdot \boldsymbol{V}=0$ in mind, we have

$$
\begin{aligned}
\frac{\partial}{\partial t} K^{\prime} & +\frac{\partial}{\partial x}\left(U K^{\prime}\right)+\frac{\partial}{\partial y}\left(V K^{\prime}\right)+u^{2} \frac{\partial U}{\partial x} \\
& +u v\left(\frac{\partial U}{\partial y}+\frac{\partial V}{\partial x}\right)+v^{2} \frac{\partial V}{\partial y} \\
= & -\frac{\partial}{\partial x}\left(u \phi^{\prime}\right)-\frac{\partial}{\partial y}\left(v \phi^{\prime}\right)
\end{aligned}
$$

where third-order terms of $u, v$ are neglected.

After integration of (7.4) in whole domain, we obtain

$$
\frac{\partial}{\partial t}\left\langle K^{\prime}\right\rangle+\left\langle u^{2} \frac{\partial U}{\partial x}+u v\left(\frac{\partial U}{\partial y}+\frac{\partial V}{\partial x}\right)+v^{2} \frac{\partial V}{\partial y}\right\rangle=0
$$

where notation $\langle>$ means space integration over hemisphere, i.e.,

$$
\langle A\rangle \equiv \iint_{D} A d x d y
$$

This expression (7.5) is a generalized form of an ordinary energy equation in barotrodic process, that is,

$$
\frac{\partial}{\partial t} K^{\prime}+\int_{y_{1}}^{y_{2}} \overline{u v} \frac{\partial U}{\partial y} d y=0 .
$$

The equation (7.5) shows that the kinetic energy of the disturbance comes partly from the acceleration of the basic current whose origin is the external forces.

On the other hand, making $U \times(7.1)+V \times$ (7.2), we have

$$
\begin{aligned}
\frac{\partial}{\partial t} \tilde{K} & +\frac{\partial}{\partial x}(U \tilde{K})+\frac{\partial}{\partial y}(V \tilde{K}) \\
& +\left(\frac{\partial V}{\partial x}-\frac{\partial U}{\partial y}\right)(V u-U v)-u^{2} \frac{\partial U}{\partial x} \\
& -u v\left(\frac{\partial U}{\partial y}+\frac{\partial V}{\partial x}\right)-v^{2} \frac{\partial V}{\partial y}+f(V u-U v) \\
= & -\frac{\partial}{\partial x}\left(U \phi^{\prime}\right)-\frac{\partial}{\partial y}\left(V \phi^{\prime}\right)
\end{aligned}
$$

Then, it turns out to be, by integration

$$
\begin{aligned}
\frac{\partial}{\partial t}\langle\tilde{K}\rangle & +\frac{\partial}{\partial t}\left\langle K^{\prime}\right\rangle \\
& \cdot\left\langle\left(\frac{\partial V}{\partial x}-\frac{\partial U}{\partial y}+f\right)(V u-U v)\right\rangle=0 .
\end{aligned}
$$

Since $(\partial V / \partial x-\partial U / \partial y+f)$ is absolute vorticity of basic field, we can rewrite (7.7) in symbolic form

$$
\frac{\partial}{\partial t}\left\langle K^{*}\right\rangle=\langle\eta(\boldsymbol{V} \times \boldsymbol{v})\rangle
$$

where $\boldsymbol{V} \times \boldsymbol{v}$ denotes $z$-component of vector product of $\boldsymbol{V}=(U, V)$ and $\boldsymbol{v}=(u, v)$.

The physical meaning of equation (7.8) might be interpreted as follows:

Since the right hand side of equation (7.8), $\langle\eta(\boldsymbol{V} \times \boldsymbol{v})\rangle$, comes from the existence of the external forcing function $\boldsymbol{F}$ in the equation of motion (2.1), the total kinetic energy $K^{*}$ may increase or decrease under the effect of external forces, and its time change is determined by the horizontal distribution of absolute vorticity $\eta$ of the basic flow and the phase of the disturbances. 
$k=1$

$a=3$

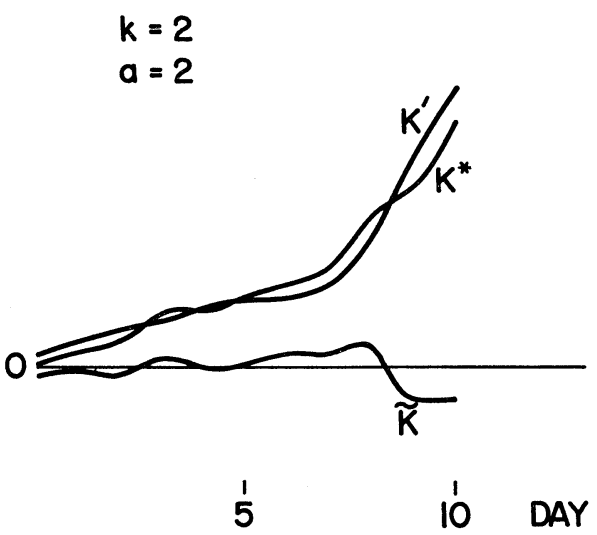

(a)

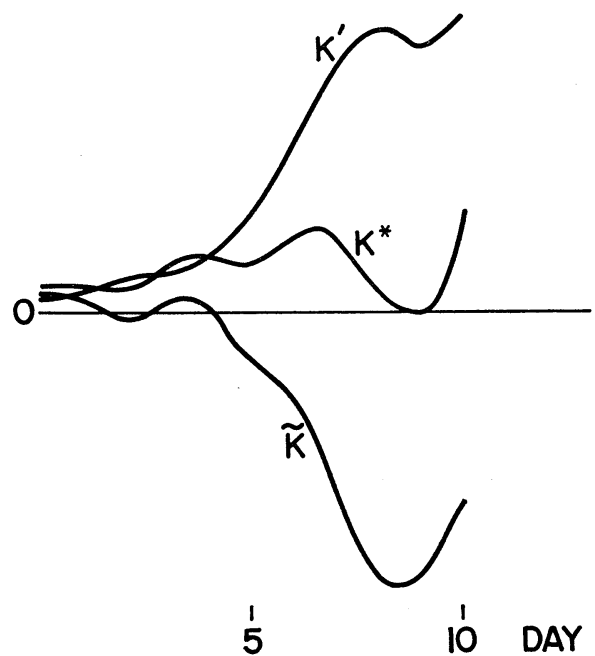

(b)

Fig. 13. Time changes of kinetic energy $K^{\prime}, K$, and $K^{*}$.

When $K^{*}$ does not increase with time, a pure exchange of kinetic energy between zonal flow and the disturbances is taking place. The breakdown does not occur in this case.

The time changes of the three forms of kinetic energy discussed in section 5 are shown in Fig. 13a and Fig. 13b. In the case of $k=2, K^{*}$ as well as $K^{\prime}$ increases with time, while, in the case of $k=1$, however, $K^{*}$ does not increase so much.

Thus we can distinguish the two types of instability ;

In the first case, the source of the kinetic energy of perturbations is the external forces. The increase of the kinetic energy of perturbations $K^{\prime}$ is accompanied with the increase of total kinetic energy $K^{*}$. The increase of the total kinetic energy $K^{*}$ will give rise to the breakdown. The transfer of kinetic energy from the basic flow to the perturbations, i.e., from $\bar{K}$ to $K^{\prime}$ through $\tilde{K}$, is rather weak in this case.

On the contrary, in the second case, kinetic energy is transfered from the basic flow to the perturbations. In this case the increase of kinetic energy of the perturbations is accompanied with the decrease of the interaction part $\tilde{K}$, while total kinetic energy $K^{*}$ is nearly conserved, and therefore the drastic variation of the flow pattern can not be expected as much as in the former case.

Now, back to the equation (7.8), we try to calculate the right hand side $\langle\eta(\boldsymbol{V} \times \boldsymbol{v})\rangle$. For the sake of simplicity, we shall make following assumptions;

Stream function of the basic flow is written as

$$
\begin{aligned}
\psi & =\bar{\psi}(y)+\tilde{\phi} \\
& =\bar{\phi}(y)-\bar{\phi}_{y} \sin k x .
\end{aligned}
$$

Then relative vorticity becomes

$$
\zeta=\nabla^{2}(\bar{\phi}+\tilde{\phi})=\bar{\phi}_{y y}-\bar{\phi}_{y y y} \sin k x+k^{2} \bar{\phi}_{y} \sin k x .
$$

Coriolis parameter is the function of only $y$, i.e.,

$$
f=f(y) .
$$

We assume that the stream function perturbation has a phase difference from that of the basic flow, i.e.,

$$
\varphi=\sin k(x+\theta) \cdot \sin l y
$$

Therefore, the right hand side of equation (7.8) becomes as

$$
\langle\eta(\boldsymbol{V} \times \boldsymbol{v})\rangle=\iint(\bar{\zeta}+\tilde{\zeta}+f) \times
$$


$\times\left[\bar{\phi}_{y} k \cos k x \cdot l \sin k(x+\theta) \cos l y\right.$

$\left.-\left(\bar{\phi}_{y}+\bar{\phi}_{y y} \sin k x\right) k \cos k(x+\theta) \sin l y\right] d x d y$.

After carrying out the integration of (7.9) with respect to $x$ over the interval $[0,2 \pi]$ and keeping the orthogonality of harmocics in mind, finally we have

$$
\langle\eta(\boldsymbol{V} \times \boldsymbol{v})\rangle=\left(I_{1}+I_{2}\right) \pi \cdot \sin \mathrm{k} \theta
$$

where

$$
\begin{aligned}
& I_{1}=\int(\bar{\zeta}+f)\left[\bar{\phi}_{y} k l \cos l y+\bar{\phi}_{y y} k \sin l y\right] d y \\
& I_{2}=\int\left(-\bar{\phi}_{y y y}+k^{2} \bar{\psi}_{y}\right) \bar{\phi}_{y} k \sin l y d y .
\end{aligned}
$$

Since the integral $I_{1}+I_{2}$ does not includ the phase angle $\theta$, the absolute value of $\langle\eta(\boldsymbol{V} \times \boldsymbol{v})\rangle$ becomes maximum when $\sin k \theta=1$ i.e.,

$$
k \theta=\frac{\pi}{2} \text { or } \theta=\frac{\pi}{2 k}=\frac{L}{4} \quad(L: \text { wave length }) .
$$

Thus the necessary condition for the maximum growth of total kinetic energy, $\partial / \partial t\left\langle K^{*}\right\rangle$, is that the phase of the disturbance is $\pi / 2 k$ different from that of the basic flow.

\section{Coclusions}

Examination of the stability properties of the stratospheric polar vortex is made by use of the barotropic vorticity equation and energy equation.

Conclusions obtained in this study are summarized as follows :

(1) Circular symmetric polar vortex is dynamically stable. The breakdown would not occur by ordinary barotropic instability, which has been treated with respect to the zonal type basic flow.

(2) When the standing waves caused by some external forces are added to the deformation of the basic flow, barotropic instability due to the deformed vortex appears. The larger the amplitude of forced waves, the more rapidly the unstable waves grow.

(3) The most unstable waves can be computed as the time integration of the perturbation equation in an initial value problem starting from a random distribution of disturbances. The pattern thus obtained is interpreted as a manifestation of the preferred perturbation with the largest growth rate. The results of the numerical calculation show a tendency for a breakdown in the case of basic flow with wave number 2 .

(4) Comparison of stability properties between the northern and southern hemispheric polar vortex is made. The polar vortex of the northern hemispheric type with wave number 2 is shown to be more unstable than the type with wave number 1 . This is in agreement with the observations of the actual atmosphere. This fact is partially explained by the difference in horizontal distribution of absolute vorticity.

(5) Energy equations corresponding to our model show that the kinetic energy of the unstable wave is partly from the basic current and partly from the external forces. The kinetic energy of the total system increases when the phase of the disturbances is $\pi / 2 k$ different from that of the basic flow.

\section{Acknowledgments}

The present author wishes to express his sincere thanks to Prof. S. Syōno who has given him the kind guidance throughout this work, and also to Dr. M. Yanai of Tokyo University, Dr. T. Matsuno of Kyūshū University and Dr. K. Miyakoda of GFDL, ESSA, for their kind advices and suggestions. Thanks are also due to Mrs. I. Akamatsu and Miss Y. Ona for their assistance. This work is partially supported by Funds for Scientific Research from the Ministry of Education.

\section{References}

Charney, J. G. and M. E. Stern, 1962: On the stability of internal baroclinic jets in a rotating atmosphere. J. atmos. Sci., 19, 159-172.

Fleagle, R. G., 1958: Inferences concerning the dynamics of the mesosphere. J. geophys. Res., 63, 137-146.

Kuo, H. L., 1949: Dynamic stability of two dimensional non-divergent flow in a barotropic atmosphere. J. Meteor., 6, 105-122.

Matsuno, T. and I. Hirota, 1966: On the dynamical stability of polar vortex in wintertime. $J$. meteor. Soc. Japan. 44, 122-128.

Miyakoda, K., 1963: Some characteristic features of winter circulation in the troposphere and lower stratosphere. Dept. Geophys. Sci., Univ. of Chicago. $93 \mathrm{pp}$. 
Murray, F. W., 1960: Dynamic stability in the stratosphere. J. geophys. Res., 65, 3273-3305.

Murakami, T., 1965: Energy cycle of the stratospheric warming in early 1958. J. meteor. Soc. Japan. 43, 262-283.

Reed, R. J., J. L. Wolfe and H. Nishimoto, 1963 : A spectral analysis of the energetics of the stratospheric sudden warming of early 1957. J. atmos. Sci., 20, 256-275.
Scherhag, R., 1952: Die explosionsartigen Stratosphärenerwärmungen des Spätwinters 1951-1952. Ber. Deutsch. Wetterdienst, 6, 51-63.

Sheppard, P. A., 1959: Dynamics of the upper atmosphere. J. geophys. Res., 64, 2116-2121.

Zudanov, L. A., 1965: The behaviour of the antarctic stratospheric warming phenomenon in the spring of 1964. International antarctic analysis center Tech. Rept. No. 4. 13 pp.

\title{
成層圏における Polar Vortex の力学的安定性
}

\author{
廣田勇 \\ (東京大学理学部地球物理学教室)
}

成層圈飞和ける周極渦（Polar Vortex）は，大規模な外力の影響によって，しばしば变形を受けるが，その变形し た渦の力学的安定性を順圧渦度方程式及びェネルギー方程式を用いて調べた。

摄動方程式を初期值問題として数值時間積分とより解いた結果, 变形した渦に対応する順圧不安定が存在して, 变 形の程度が大きいほど, より不安定な擾乱が発達することがわかった。

Volar Vortex の変形の様相は，南北両半球に扔いて異っているが，その両者飞対応する変形渦（波数 1 と 2 ）を 数値モデルで構成し, その安定性を比較した結果, 北半球に対応する波数 2 の变形渦のほうがより不安定であり, 急 激な崩壊現象（Breakdown）の起り得ることが示された。

一方このモデルに関する運動エネルギー方程式を作ってみると，外力との相互作用が，系全体の運動エネルギーの 增減に関与し，擾乱と変形渦の位相が 4 分の 1 波長異っているときと，最大エネルギー増加が期待される。

これらの種々の力学的特性は, 実測の結果と比較して, 定性的によく一致している。 